\title{
Photoionisation effects in hydrodynamical simulations of planetary nebulae
}

\author{
L. Hernández-Martínez, D. Estrella, P. F. Velázquez, \\ A. Esquivel and A. C. Raga
}

Instituto de Ciencias Nucleares, Universidad Nacional Autónoma de México, 70-543, México, D. F. México

email: lilihe.mtz@gmail.com

\begin{abstract}
We explored the photoionisation effects on both the proper motion and emission of planetary nebulae NGC 6302, by means of hydrodynamical simulations. We used the GUACHO code, which includes the photoionisation due to central source (Esquivel et al. 2009, Esquivel \& Raga 2013). We model these PNe considering an interacting stellar fast wind with and ejected toroidally shaped slow wind (Uscanga et al. 2014). Synthetic $\mathrm{H} \alpha$ emission maps were obtained from our numerical results in order to do a comparison between the cases with and without photoionisation. Using a wavelets fittering method on our results for the ionisation case, we do not find an increase in the proper motion velocities, however we can see an accelerated expansion in both cases. For the ionisation case the $\mathrm{H} \alpha$ emission presents an increase.
\end{abstract}

Keywords. Planetary Nebulae, Bipolarity, Hydrodinamics

\section{Introduction}

NGC6302 is a butterfly-shaped bipolar Planetary Nebulae (PN) with a very cumply structure and a central dense circumstellar disc. The lobes of this PN present an accelerated expansion (also known as Hubble-type expansion) maybe produced by a binary system (Szyszka et al. 2011). We used GUACHO to model NGC 6302 and reproduce the PN lobes. GUACHO is a massive parallel 3 dimensional hydrodynamical code with uniform mesh and that can take into account Ly $\alpha$ photons for the photoionisation prescriptions (Esquivel et al. 2009, Esquivel \& Raga 2013).

\section{Hydrodynamical simulations}

We have performed hydrodynamical simulations with the GUACHO code and study the effects of the photoionisation on the proper movement of the clumpy structure shown in the $\mathrm{H} \alpha$ emission maps. The computational domain is $256 \mathrm{x} 256 \mathrm{x} 512 \mathrm{cells}(0.5 \mathrm{x} 0.5 \mathrm{x} 1 \mathrm{pc})$ in the uniform mesh, yielding a resolution of $0.002 \mathrm{pc}$. We include two winds, following the model presented in Uscanga et al. (2014). The physical parameters of these winds are: a) for the slow wind we used $\dot{\mathrm{M}}_{S}=5 \times 10^{-4} \mathrm{M}_{\odot} \mathrm{yr}^{-1}, \mathrm{~T}_{S}=10^{3} \mathrm{~K}, \mathrm{~V}_{S}=15 \mathrm{~km} \mathrm{~s}^{-1}$ and b) for the fast wind we used $\dot{\mathrm{M}}_{F}=1 \times 10^{-7} \mathrm{M}_{\odot} \mathrm{yr}^{-1}, \mathrm{~T}_{F}=10^{6} \mathrm{~K}, \mathrm{~V}_{F}=1800 \mathrm{~km} \mathrm{~s}^{-1}$. We ran the simulations by $2000 \mathrm{yr}$, and we focus on the last $10 \mathrm{yr}$ of the PN evolution in order to compare with the observations. In this simulations we imposed a turbulent density distribution (Esquivel et al. 2003) an ionising flux of photons of $10^{46} \mathrm{erg} \mathrm{s}^{-1}$ divided in $10^{5}$ rays, emulating the diffuse radiation of the system. Our simulations show the density structure and the morphology like as NGC 6302 (size and structure). However, the $\mathrm{H} \alpha$ image with photons indicate us a clearly increase in the emission of the clumps. In order to study the dynamical of our numerical simulations, we processed our $\mathrm{H} \alpha$ maps (see 


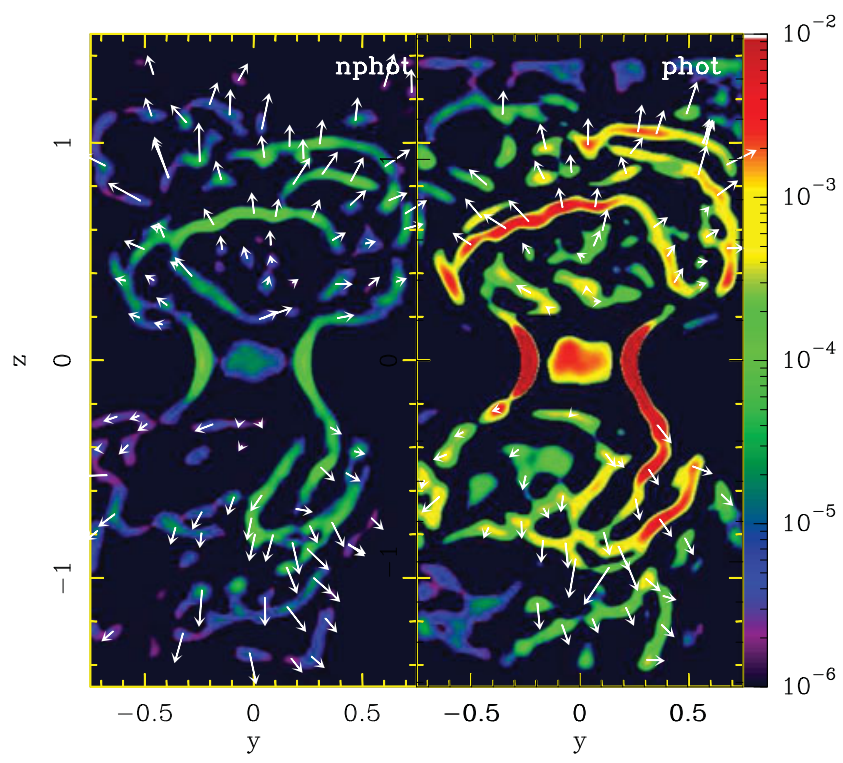

Figure 1. Synthetic $\mathrm{H} \alpha$ images of our numerical simulations, convolved with a wavelet function with sigma $=5$. To obtain the proper motions we followed the method described in Raga et al.(2016) with synthetic images that have 10yr between each other, arrows depict the proper motions.

Figure 1) with the wavelets technique presented in Raga et al. (2016), i.e. we convolved the synthetic images by using a Mexican-hat function with a given sigma, in our case was of 5 sigma. To obtain the proper motions we followed the method described in Raga et al. (2016) with synthetic images that have $10 \mathrm{yr}$ between each other, arrows depict the proper motions. Then we compared with HST observations (which are going to be presented in Hernández-Martínez et al. in prep.)

\section{Discussion and future work}

We reproduced the Uscanga et al. 2014 model using GUACHO code which includes a central ionising source. We have compared the proper motion obtained from numerical results including or not the photoionisation of a central sourde. We can not discriminate between the two different data. So that, for our simulations the photoionisation apparently does not has an important effect in the proper motion. However, we can see a Hubble-type expansion, as it was reported by Szyszka et al. (2011). We also see an increase in the $\mathrm{H} \alpha$ emission in the simulation with photoionisation. These are preliminary results and we are still working on it.

\section{References}

Esquivel, A., Cantó, J., \& Rodríguez-González, A. 2009, A\& A, 507,855

Esquivel, A., Lazarian, A., Pogosyan, P., \& Chou, 2003, MNRAS, 342, 325

Esquivel\& Raga 2013, ApJ, 779,111

Hernández-Martínez et al. in prep.

Raga, A. C., Reipurth, B., Esquivel, A., \& Bally, J. 2016, AJ, 151, 113

Szvszka, C., Zijlstra, A. A., \& Walsh, J. R. 2011, MNRAS, 416, 715

Uscanga, L., Velazquez, P. F., Esquivel, A., Raga, A. C., \& Boumis, P. 2014, MNRAS, 442, 3162 